\title{
Lesen und verstehen
}

\section{Interkulturalität und interkulturelle Kommunikation}

\author{
in und mit Texten des Mittelalters
}

\section{Claudia Brinker-von der Heyde}

\begin{abstract}
Since the middle of the 2oth century, German Medieval Studies has been confronting the nationally oriented field of German Studies with an intercultural approach to textual analysis - after all, its objects of interest proved culturally unfamiliar in all respects, even though stemming from ones own culture. Scholarship in interculturality, understood as a project, does not put emphasis on the Other and the Foreign, as does the concept of alterity; rather, it deals with texts in a processual and open-ended manner. As its method, one might suggest a kind of intercultural dialog with the artifacts that aims at subverting the delimitations between the >Own< and the >Foreign<. Following Bhatti and Kimmich, this article proposes similarity as a structural principle, as the concept of similarity does not negate differences, but repeals the binary dichotomy of Own and Foreign, thereby opening the perspective on commenness and interweavings.
\end{abstract}

Title: Reading and Understanding. Interculturality and Intercultural Communication in and with Medieval Literature

Keywords: medieval literature; intercultural communication; similarity as structural principle; methodology; interculturality as project

Interkulturalität ist seit den 8oer Jahren des letzten Jahrhunderts im Wissenschaftsdiskurs verschiedenster Disziplinen omnipräsent, nicht zuletzt deshalb, weil sie sich als »vielfach einsetzbarer und deutungsoffener Begriff « mit den »Themen, Fragestellungen und Methoden «(Gutjahr 2010: 17) verbinden lässt, die vergangene und gegenwärtige Verhältnisse von Kulturen unter dem Postulat absoluter Gleichwertigkeit in den Blick nehmen (vgl. Yousefi/Braun 2011: 29). Ziel war und ist es, deutlich zu machen, dass - anders als von einer nationalstaatlichen Geschichts- und oft auch Literaturgeschichtsschreibung behauptet Kulturen keine holistischen Gebilde sind, keine gewachsenen oder gewordenen homogenen Einheiten oder gar Entitäten, sondern immer geprägt sind von Einflüssen anderer Kulturen, sei es durch Migration, Kolonialismus, politische und gesellschaftliche Kontakte, Handel, Reisen u.v.a.m. Deshalb sind nahezu alle Gesellschaften dieser Welt heute als »kulturelle Mischlinge« (Flechsig 2001: 3) anzusehen, mit einer »patchwork-Identität« (Welsch 2010: 46). D.h., dass die kulturelle Identität einer Gruppe oder eines Individuums nicht auf ein Bezugssystem begrenzt bleibt, mithin also auch nicht von der kulturellen Identität, sondern von kulturellen Identitäten gesprochen werden muss. Immer wieder ist in 
der Forschung zu lesen, dass in den modernen, multikulturellen Gesellschaften interkulturelle Kompetenz zwingend erforderlich ist, nicht zuletzt, um einem »clash of civilizations«, wie ihn Huntington (1996) heraufbeschwört, keinen Raum zu geben, sondern ohne Vorurteile kulturelle Vielfalt bewusst und aktiv zu leben. Allerdings wird in den einzelnen Forschungsansätzen und -thesen häufig der Kulturbegriff, der diesen zugrunde liegt, wenig oder gar nicht offengelegt (vgl. Földes 2007; Bhatti/Kimmich 2015), obwohl Kultur - so Hart-Nibbrig (1999: 95) - »begrifflich ein diffuses >Ganzes< und von der zähen Dehnbarkeit eines Hosenträgers « ist. Aber selbst dort, wo die Überlegungen explizit auf einem erweiterten Kulturbegriff beruhen, der »alles umfaßt, was an Menschengeschichte innerhalb der Naturgeschichte geschehen ist und geschieht « (Marschall 1999: 21f.), werden implizit doch mit den Begriffen >Multikulturalität< und $>$ Interkulturalität< häufig nur die Kategorien (geographische) Herkunft/Nationalität, Ethnie und - äußerst aktuell - die Zugehörigkeit zu einer der drei Buchreligionen - Islam, Christentum, Judentum - assoziiert, ohne deren jeweilige Binnendifferenzierungen zu berücksichtigen. Interkulturelle Vielfalt umgreift aber alle nur denkbaren menschlichen Lebensbereiche. Die »Charta der Vielfalt «, die seit 2007 von zahlreichen Unternehmen und Institutionen unterzeichnet wurde, setzt es sich denn auch zum Ziel, dass alle Mitarbeiter und Mitarbeiterinnen eines Unternehmens »Wertschätzung erfahren [sollen] - unabhängig von Geschlecht, Nationalität, ethnischer Herkunft, Religion oder Weltanschauung, Behinderung, Alter, sexueller Orientierung und Identität« (Ridder/Jorzik 2012: 5). Das dazu entwickelte >Rad der Diversität< (vgl. Abb. 1) $)^{1}$ scheint mir bestens geeignet, um Verschmelzungen, Ähnlichkeiten und Differenzen zwischen Kulturen und kulturellen Zugehörigkeiten zu zeigen. Denn je nachdem, welche Kategorien und Dimensionen zueinander in Verbindung gesetzt werden - Religion, Geschlecht, Beruf, Nationalität, Ethnizität, Wissensordnungen, Bildung, Sprache, Kunst usw. -, verändern sich kulturelle Identitäten bzw. Eigen- und Fremdwahrnehmungen von Individuen. Ein Herzspezialist z.B. mag zwar nicht die Sprache einer Fachkollegin verstehen, er mag einer anderen Ethnie, einer anderen Nation, einem anderen Glauben angehören, und doch wird er im Fachgespräch mehr Gemeinsamkeiten zu dieser finden als in der Kommunikation mit z.B. einem Bergbauern derselben Ethnie, derselben Sprache, derselben Nation und demselben Glauben. Dennoch betonen nahezu alle Forschungsarbeiten, dass ein »Wissen um die eigene Kulturalität« sowie um den »mentalen Abstandsraum zwischen den Kulturen« unabdingbar sei (Gutjahr 2010: 19), obwohl Földes bereits 2007 betont hatte, dass sich Menschen unterschiedlicher Kulturen »zugleich sehr ähnlich und sehr unterschiedlich« sind (Földes 2007: 17). Ein gewisser und bisher keineswegs gelöster Widerspruch wird hier deutlich: Obwohl die Existenz einer kulturellen Einheit dem Konzept der Inter- und noch mehr der Transkulturalität eigentlich entgegenstehen, bleibt diese implizit doch wei-

1 Die organisatorischen Dimensionen beziehen sich dabei auf die jeweilige Organisation und sind hier vernachlässigbar. Für unser Thema entscheidend sind die inneren und äußeren Dimensionen. 
terhin mitgedacht, denn nach wie vor ist meist von Beziehungen zwischen verschiedenen Kulturen die Rede, was bedeutet, dass doch klar bestimmbare Kulturgrenzen und Kulturunterschiede von vornherein unterstellt werden (vgl. Gutjahr 2010: 25). Damit rückt die interne Diversität (vgl. Senghaas 1998: 139f.; Yousefi/Braun 2011: 25) einer jeden Kultur in den Hintergrund und Interkulturalität wird gerade nicht als »eine Realität, die in allen Gesellschaften seit Menschengedenken anzutreffen ist« (ebd.: 27), wahrgenommen.

Abbildung 1: Charta der Vielfalt (Ridder/Jorzik 2012: 15).

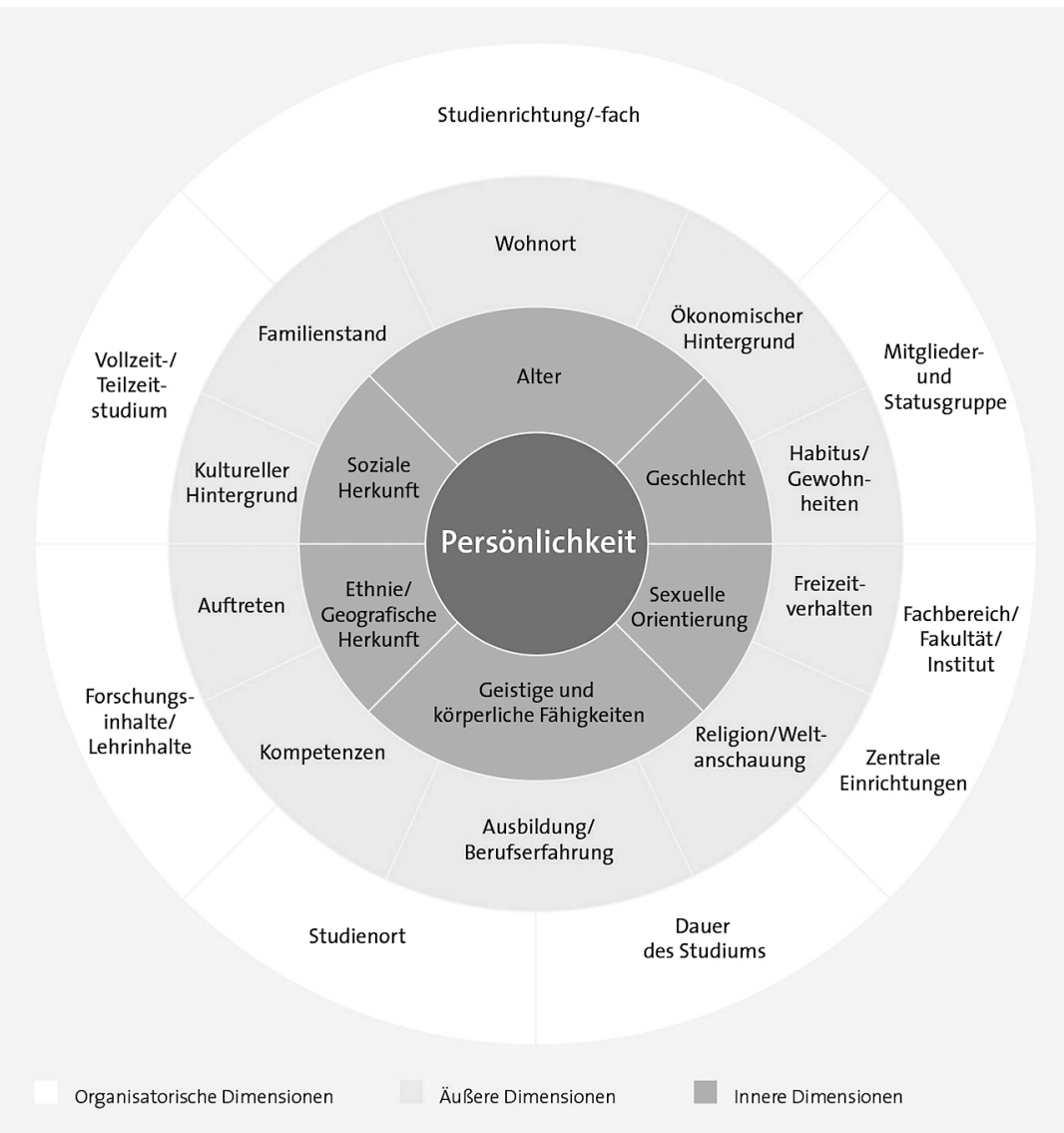

Interkulturalität wird also zumindest unterschwellig nach wie vor in erster Linie mit einer Begegnung von Menschen unterschiedlicher Herkunft und Sprache verknüpft. D.h., trotz aller Versuche, den Kulturbegriff zu erweitern und prinzipiell offenzuhalten, sind immer noch diese beiden Kategorien zentral. Und auch die Prämisse, dass die eigene Kultur über Tradition identitätsstiftend sei, hält sich hartnäckig, ungeachtet des Wissens darum, dass solche Traditionen häufig ganz bewusst behauptet und gesetzt wurden und werden, um sie zu politischen Zwecken zu nutzen. Das Mittelalter ist zweifellos eine Projektionsfläche für derartige 
politische, gesellschaftliche Instrumentalisierungen. Vor allem im 19., aber auch noch im 2o. Jahrhundert wurden Geschichts- und Kulturmythen geschaffen, die sehr viel wirkmächtiger waren als ein wie auch immer geartetes >reales< Mittelalter. Bis heute haben sie ihre Wirkung nicht verloren und prägen nach wie vor das Bild vom Mittelalter. Exemplarisch zu nennen seien hier Novalis' Vision eines einigen christlichen Mittelalters (vgl. Novalis 1986), die u.a. von Herder entwickelte und bis weitins 20. Jahrhundert kolportierte Vorstellung, dass ein Volk seine Wurzeln in einer homogenen, gemeinsamen Ethnie, Religion und Sprache hat und daraus auch seine Legitimation erhält (vgl. Herder 1878; 1891), ${ }^{2}$ die ebenfalls von Herder geprägte Idee einer Volksseele (Herder 1878: 27; 1891: 185, 201 u.ö.), ${ }^{3}$ die Etablierung des Nibelungenlieds als Nationalepos im 19. Jahrhundert (vgl. Schlegel 1965: 109-114) sowie seine agitatorisch-politische Verwendung etwa in Form der > Dolchstoßlegende< oder in der von den Nationalsozialisten propagierten > Nibelungentreue < und in Görings berühmt-berüchtigter Stalingradrede zum zehnten Jahrestag der Machtergreifung, in der er den Kampf der Soldaten in Stalingrad mit demjenigen der Nibelungenhelden vergleicht und ihren Untergang glorifiziert. Aber auch in filmischen Mittelalteradaptionen halten sich hartnäckig verklärte Mittelalterbilder des 19. Jahrhunderts und werden bombastisch inszeniert.

Unter drei unterschiedlichen Blickwinkeln soll deshalb im Folgenden überlegt werden,

1. ob Interkulturalität als Theorie wie als Methode geeignet ist, um derart klischierten Bildern des Mittelalters entgegenzuwirken,

2. ob bzw., wenn ja, wie interkulturelle Fragestellungen und Zugänge helfen, Artefakte des Mittelalters - in unserem Fall vor allem literarische Texte - zu verstehen, und

3. ob damit interkulturelle Kompetenzen erworben werden können, die auch im Gegenwartsdiskurs und in interkulturellen Begegnungen fruchtbar eingesetzt werden können.

\section{INTERKULtURALITÄt In DER MediäVISTIK: vom Paradigma zum Projekt!}

Die nicht zuletzt politische Gefahr, die von einer kulturellen Ab- und Ausgrenzung oder einem kulturellen Überlegenheitsdenken ausgeht, hat die in ihren Anfängen zweifellos kulturhistorisch konzipierte Germanistik im 19. und in

2 | Noch 1966 geht Leo Weisgerber von einer generellen Einsprachigkeit des Menschen aus und davon, "daß die geistige Anverwandlung der Welt die Geschlossenheit einer Muttersprache erfordert" (Weisgerber 1966: 285).

3 | Vgl. z.B.: „In jedem Bardenlied zeigt sich ein Volk, dessen Seele ganz der Tapferkeit und einer feierlichen Liebe flammte" (Herder 1878: 27). Oder er spricht von der "Seele des Volks, die doch nur fast sinnlicher Verstand und Einbildung ist" (Herder 1891: 185) sowie von der "Seele der alten, wilden Völker" (ebd.: 201). 
der ersten Hälfte des 20. Jahrhunderts drastisch gezeigt, als sie sich auf nationale Legitimierungen fokussierte, programmatisch »den Metamorphosen in der Identität des deutschen Geistes « widmete (Schmitz 1991: 172) und dabei das Mittelalter zur Projektionsfläche einer nationalen, kollektiven, auf Traditionen beruhenden kulturellen Identität machte. Dem setzte in der zweiten Hälfte des 20. Jahrhunderts die Mediävistik Interkulturalität als Paradigma entgegen, auch wenn der Begriff dafür am Anfang noch fehlte (vgl. Sieburg 2011: 11). Denn sie erkannte nun, dass sie sich mit Texten auseinanderzusetzen hatte, die in jeder Hinsicht - Sprache, Werte und Normen, politische und gesellschaftliche Systeme, Poetik, literarische Gattungen, Themen, Motive usw. - fremdkulturell erscheinen und doch gleichzeitig Teil unserer Kultur sind (vgl. Schumacher 2010: 49; Kasten/Bachorski/Kugler 1997; Sieburg 2011). Damit ist die mediävistische Forschung prädestiniert, »die immanente Interkulturalität der sogenannten deutschen Nationalkultur wieder sichtbar zu machen « (Schmitz 1991: 172) und eine historisch gewachsene, nationale, kulturelle Einheit als Konstrukt und Projektion neuzeitlicher Jahrhunderte zu entlarven. Anders als das, seit den $1970 e r$ Jahren in der Mediävistik gängige, fast schon >Zauberwort< Alterität, das vor allem die Eigenart, das Fremde, das Irritierende des Mittelalters gegenüber der Moderne betont und das noch 2012 im Titel eines Sammelbandes »Leitkonzept für historisches Interpretieren« genannt wird (Becker/Mohr 2012), legt das Paradigma Interkulturalität den Fokus gerade nicht nur auf das Fremde, sondern genauso auf Konstanten als ein »Korrektiv«, durch das »Strukturen der longue durée sichtbar« werden können (Braun 2013: 27f.), und blickt vorurteilsfrei auf die fremdgewordene Vergangenheit und ihre Texte, so dass diese nicht einfach als längst vergangene, exotische Welten oder als befremdende Negativexempel wahrgenommen werden, sondern nach Wegen zum Verstehen des fremden Eigenen gesucht wird. Denn - dies ist das Credo von Interkulturalität -: »Ignoranz ist kein interkulturell tragfähiges Konzept« (Herweg 2017: 13). Weil Alterität aber immer vom eigenen Standpunkt ausgeht und nur das Gegenüber, nicht sich selbst als das Andere, sieht, ist Ignoranz oder zumindest Verständnislosigkeit durchaus eine Gefahr. Demgegenüber vermeidet ein interkultureller Zugang zu den Artefakten, allen voran den Texten, voreilige normative Wertungen, weil das Eigene nicht a priori die Norm und das Andere nicht a priori eine Abweichung bedeutet. Dies gilt umso mehr, wenn auch in der Mediävistik, wie Heimböckel und Weinberg (vgl. 2014) es für die Neugermanistik vorschlagen, Interkulturalität nicht mehr zu einem statischen Paradigma erklärt wird, sondern vielmehr zu einem Projekt, das prozesshaft, zeitlich entgrenzt und in seinem Ergebnis »ganz und gar offen « bleibt (ebd.: 122), ${ }^{4}$ was immer auch »die Möglichkeit des Nichtzustandekommens, des Scheiterns, des Aufgebens [...] mit im Visier « hat (Stanitzek 1987: 138; vgl. Heimböckel/Weinberg 2014: 123). Zentraler Begriff im Rahmen von Interkulturalität ist für Heimböckel und Weinberg das Staunen als eine Form »bewußtwerdenden Nichtwissens« (Guzzoni 2012:

4 | Heimböckel/Weinberg (vgl. 2014: 122) verwenden dabei den Begriff "Projekt" explizit in der "klassischen "Semantik des 17. Jahrhunderts. 
12; vgl. Heimböckel/Weinberg 2014: 121). Denn ein solches Staunen durchbricht »die Selbstverständlichkeit eigener kultureller Erfahrungen« (Schlesier 1996: 55; vgl. Heimböckel/Weinberg 2014: 121), stellt »interkulturelle Grundkategorien wie das Eigene und Fremde in Frage« (ebd.), führt damit zu Verunsicherungen und - ganz entscheidend - zu Verschiebungen von Grenzen (vgl. ebd.: 120, 124 u.ö.).

Methodisch bietet sich für einen solch projekthaften interkulturellen $\mathrm{Zu}$ gang zu dem allmählichen Verstehen, aber auch zum möglichen Eingeständnis des Unverstehbaren von vergangenen (Text-)Welten, die interkulturelle Kommunikation an.

\section{Interkulturelle Kommunikation als Methode}

»Interkultureller Dialog beginnt mit dem Wahrnehmen der Differenzen innerhalb der Kulturen selbst«, so Roman Herzog anlässlich der Verleihung der Goethe-Medaillen am 22. März 1998 in Weimar (Herzog 1998). Roman Herzog dachte dabei sicher an eine Wahrnehmung auf synchroner Ebene, diese kann und - wie ich zeigen möchte - muss aber auch und gerade auf der diachronen Ebene geschehen, weil mit einem grundsätzlich pluralistisch angelegten interkulturellen Dialog (vgl. Yousefi/Braun 2011: 39) mit längst Vergangenem das vermeintlich unhintergehbar Eigene - wie etwa eine homogene >nationale< Geschichte bzw. >nationale< Identität - zu wackeln beginnt und Fremdes und Eigenes in eine neue Beziehung zueinander treten. D.h., es geschieht genau die Verschiebung und Verunsicherung, die nach Heimböckel und Weinberg (vgl. 2014) das Projekt Interkulturalität auszeichnet.

Gelingen kann ein solch diachrones Wahrnehmen wenn auch nicht durch einen »unmittelbaren Dialog« (Sieburg 2011: 16), so doch durch einen mittelbaren, nämlich durch einen Dialog mit Artefakten einer fremd anmutenden seigenen< Vergangenheit. Wie in jedem interkulturellen Dialog, darf es aber nicht bei bloßer Wahrnehmung des Fremdgewordenen bleiben, sondern es geht darum, den dinglichen Dialogpartner (vgl. Niehaus 2010) ernst zu nehmen und so weit wie möglich zu verstehen. Letzteres aber setzt voraus, dass man sich von Anfang an etwaiger Vorurteile bewusst ist, Vorannahmen ausblendet, das eigene Selbst reflektiert, Missverständnisse nicht nur einkalkuliert, sondern als durchaus produktiv begreift (vgl. Leggewie/Zifonun 2010: 16) und deshalb immer wieder neugewonnene Erkenntnisse hinterfragt, vor allem aber, dass man auf absolute Wertungen verzichtet und sich der Grenzen des Verstehens bewusst bleibt.

Jedes Verstehen, jede Annäherung an ein wie auch immer geartetes Gegenüber aber bedarf des Redens (vgl. Gutjahr 2010: 18). Unmittelbar akustisch gelingt dieses bei Artefakten der Vergangenheit ${ }^{5}$ nicht, aber im Lesen von Literatur - um die es hier gehen soll - geben wir ihnen erneut eine, nämlich unsere

5 | Wobei keineswegs nur Artefakte der Vergangenheit zum interkulturellen Dialog herausfordern, denn - dies betont Niehaus (vgl. 2010: 33) - auch der heutige Kontakt mit 
Stimme, wie dies mittelalterlichen Rezipienten ganz selbstverständlich war. Im Medium der Schrift konnte die Stimme des Dichters auch räumlich entfernt gehört werden, sei es durch Boten, Vorleser oder auch durch lautes Selbstlesen. Immer wieder thematisieren Autoren diese Medialität von Schrift und Buch.

Wis mîn zunge und mîn munt bittet das Ich in Hartmanns von Aue Klage sein kleines büechel (Hartmann von Aue 1972: 2. Bl., vv. 813 u. 811), wenn er es seiner Geliebten zusendet. Mit einem $\mathrm{Nu}$ var hin, welhischer gast schickt Thomasin von Zerclaere (1965: v. 14685) sein Buch auf die Reise in tiusche lant (ebd.: v. 86) und empfiehlt ihm, sich auf den Schoß eines tüchtigen Mannes zu setzen (vgl. ebd.: v. 14694), eine Haltung, in der bis heute Bücher gelesen werden, eine Haltung aber auch, die im Mittelalter eine Adoption rechtsgültig macht (vgl. Wenzel 1995: 210; Brinker-von der Heyde 2007: 119).

Das Buch ist damit sehr viel mehr als ein Gegenstand, der in die Hand genommen werden kann, es ist ein eigentliches Lebewesen mit einem Körper, menschlichem Verhalten und einer hörbaren Stimme.

Wer hat mich guoter ûfgetân, fragt das Buch denjenigen, der den Roman Wigalois (Wirnt von Grafenberg 2005: v. 1) aufschlägt. ${ }^{6}$ Es bietet einen nicht an Zeit und Raum gebundenen Dialog an, sollte es jemand sein, der mich kann / beidiu lesen und verstên (ebd.: vv. 2f.), der also nicht nur lesen, sondern auch verstehen will. Und es ermahnt diesen, gutwillig zu lesen, nichts zu verfälschen, worunter es doch so häufig zu leiden hat. D.h., es fordert einen interkulturellen Dialog und die Bereitschaft zu interkulturellen Erfahrungen ein, weil es nicht nur heutige, sondern auch zeitgenössische Leser und Leserinnen in eine ihnen unbekannte, fremde Welt entführt. Und weil das Buch nicht bloßes Ding, sondern Dialogpartner ist, lesen mittelalterliche Leser und Leserinnen nicht leise, sondern laut, und wiederholen, vor sich hin murmelnd, das Gelesene so lange, bis sie sich sicher sind, es verstanden zu haben. Und dazu bewegen sie sich, wie es Menschen tun, die mit einem anderen im Gespräch sind (vgl. Illich 1991: 57-62). Ohne uns dessen bewusst zu sein, geben wir auch heute noch dem Buch einen Körper, wenn wir vom Buchrücken, dem Textkörper, dem Briefkopf oder den Fußnoten sprechen. Und zumindest mittelalterliche Texte müssen auch laut gelesen werden, denn im Leise-lesen erschließt sich das Mittelhochdeutsch, die >eigene fremde< Sprache sehr viel weniger als im lauten Sprechen. ${ }^{7}$ D.h., die heute übliche Art des stummen Lesens ändert sich ganz selbstverständlich, passt sich dem Buch und seiner Kultur an. Verstanden werden die der heutigen Zeit so fremd gewordenen Texte allerdings deshalb noch nicht und auch das Wiederholen des Gelesenen allein genügt nicht mehr. Vielmehr bedarf es eines dauernden Nachfragens, und zwar nicht nur bei dem einen Buch, sondern bei vielen

anderen Kulturen beruht zu einem eher geringen Teil auf persönlichen Begegnungen, sondern wird viel häufiger über deren Medien und Artefakte hergestellt.

6 | Zum sprechenden Buch vgl. Bendheim 2017: 196-219.

7 | Die Fremdheit in der eigenen Sprache wird in der mediävistischen Interkulturalitätsforschung immer zentral herausgehoben. Vgl. Müller 2009 u. Sieburg 2011 mit weiterführenden bibliographischen Angaben. 
anderen, die Antworten auf die Fragen anbieten. Wer also mittelalterliche Literatur nicht nur liest, sondern auch verstehen will, muss sich ein weitverzweigtes Netzwerk an Texten schaffen, damit der literarische Dialog mit dem gewählten Buch gelingen kann. Man wird dabei auf sehr unterschiedliche Positionen und Meinungen stoßen, die immer wieder verunsichern und $\mathrm{zu}$ dauerndem Umdenken zwingen. Man wird bei intensiver Beschäftigung mit den Texten im zunächst Fremden häufig Ähnliches erkennen und doch immer wieder auf Grenzen des Verstehens stoßen. Dies geschieht nicht nur, weil das Denken und die Sprache unvertraut sind, sondern auch und gerade, weil Literatur nie das Abbild einer wie auch immer gearteten Realität ist, sondern subversive Gegenwelten zu dieser schafft, der Realität eine kaum je vorhandene Idealität zuspricht oder auch eine ebenso wenig realistische absolute Verworfenheit anprangern kann. Nicht zuletzt beruhen die vielen Klischeebilder vom Mittelalter, die sich bis heute so hartnäckig halten, darauf, dass literarische Texte distanzlos wie historische Quellen gelesen und damit zwangsläufig missverstanden wurden und vielerorts bis heute werden. Denn je nachdem, welche Textsorten gelesen und mit welchen Vorannahmen sie möglicherweise bereits unterlegt werden, bestätigen sich durchaus sowohl die positiven wie negativen Zuschreibungen, die man zum Mittelalter kennt: Es findet sich gleichermaßen ein goldenes wie ein finsteres Zeitalter; es finden sich Ausschweifungen, Bildungsferne und Brutalität genauso wie > Ritterlichkeit < und minnecliche Damen, denen Männer zärtliche Minnelieder sangen und für die sie bereit waren, ihr Leben zu wagen; man erfährt von gelebter Gläubigkeit und Nächstenliebe, aber auch von Ausbeutung durch selbstherrliche Kirchenmänner; von Mut, Tapferkeit, Freigebigkeit, Maßhalten, gutem Benehmen und Bescheidenheit als höfisch ideale Tugenden ist genauso die Rede wie von Selbstjustiz, Grausamkeit und Streitsucht; es wird gehasst, geliebt, intrigiert, getrauert, gefeiert - keine Emotion ist mittelalterlichen literarischen Figuren fremd. Aus dieser Vielfalt und Widersprüchlichkeit literarischer Setzungen eine homogene Vorstellung dessen zu gewinnen, was das Mittelalter war, ist kaum möglich. Aber genau dies ist seit dem Beginn der Mittelalterrezeption in der Mitte des 18. Jahrhunderts immer wieder gemacht worden, weil das Mittelalter als Projektion dienen konnte, um entweder Missstände der eigenen Zeit anzuprangern oder die eigene Fortschrittlichkeit zu betonen. Sich hartnäckig haltende Mittelalterbilder sind also Setzungen späterer Zeiten (vgl. dazu Oexle 1997). Um sie als solche zu erkennen und zu entlarven, bedarf es eines unverstellten Herangehens an die mittelalterlichen Texte als eine »Form der Arbeit am kulturellen Gedächtnis« (Gutjahr 2010: 27). ${ }^{8}$ Dafür ist der interkulturelle literarische Dialog als eine besondere Form der Interkulturalität prädestiniert. Von der Interkulturalitätsforschung ist er allerdings bisher kaum wahrgenommen worden. Auch bei Yousefi/Braun fehlt er in der auf Vollzähligkeit

8 | Dies gilt sowohl auf der Ebene des - hier lesenden - Individuums als auch auf einer Strukturebene gesellschaftlicher Kontexte und sozialer Situierung. Diese Ebenen werden im interkulturellen Dialog oft nicht genau unterschieden. Ich konzentriere mich hier auf die Ebene des Individuums bzw. des Rezipienten mittelalterlicher Texte. 
angelegten Auflistung interkultureller Dialoge (vgl. Yousefi/Braun 2011: 31). Dabei erfordert ja gerade der interkulturelle literarische Dialog besonders hohe Kompetenzen, weil er sich nicht mit dem Literalsinn begnügen kann, sondern immer überlegen muss, was sich hinter diesem an Bedeutung verbergen könnte, eine hermeneutische Fähigkeit, die letztlich für jede Form eines interkulturellen Kontakts, so er gelingen will, vonnöten wäre. Wie jeder interkulturelle Dialog schließt auch der literarische Dialog Missverständnisse nicht aus, aber das Wissen um verschiedene Möglichkeiten literarischen Verstehens und Interpretierens verhindert den Anspruch auf absolute, allgemeingültige Setzungen und toleriert damit auch unterschiedliche Wahrnehmungen (vgl. Kasten/Bachorski/ Kugler 1997: 68f.). Der literarische Dialog mit mittelalterlicher Literatur kann dabei zweierlei leisten: Zum einen hilft er, Kompetenzen zu erwerben, »die einen reflektierten Umgang mit Fremdheit, mit >Anderssein< ermöglichen « (ebd.: 66), darüber hinaus aber führt er zur Erfahrung, sich in der vermeintlich eigenen Kultur gleichermaßen fremd wie vertraut zu fühlen. Und dies in mehrfacher Hinsicht: Fremd ist die Sprache und doch als Deutsch zu erkennen, wobei der Grad der Fremdheit durchaus verschieden ist, je nachdem aus welchem deutschsprachigen Raum - dem alemannisch-bayerischen oder dem nördlichniederdeutschen - der Leser oder die Leserin stammt. Irritierend ist das Fehlen einer deutschen Hochsprache, was bewusst macht, dass es die postulierte Einheit der deutschen Sprache gar nie gegeben hat, im Gegenteil: Die Unterschiede in den einzelnen >deutschen Sprachregionen waren so groß, dass deren Sprecher sich kaum verstanden. Befremdlich ist die Dominanz einer $>$ Fremdsprache $<$ des Lateins, in der Schriftproduktion und im Bildungsdiskurs und erinnert gleichzeitig doch an die viel gescholtene heutige Lingua franca, das Englische. Fremd sind die literarischen Stoffe und doch in gewisser Weise aus der Fantasyliteratur bekannt, fremd ist die gängige Form der Schemaliteratur, die bekannte Stoffe wiedererzählt und aus einem Arsenal bekannter Bausteine schöpft, und doch ist dieses literarische Verfahren nicht nur in heutiger >Trivialliteratur< (vgl. Braun 2013: 23), sondern auch in Fernsehserien und Filmen allgegenwärtig. Fremd mutet das in den Texten entwickelte Weltbild und das Agieren der literarischen Figuren an, moralische Werte und Emotionen klingen dagegen mehr oder weniger vertraut. Fremd ist einem das, in literarischen Texten meist idealisierte, ständisch gegliederte Gesellschaftssystem, und man vergisst dabei nur allzu leicht, dass unser heutiges zwar anders, aber nicht minder hierarchisch bzw. inkludierend und exkludierend strukturiert ist. Fremd ist der Alleinherrschaftsanspruch der Christen, erstaunlich ähnlich zu islamistischfundamentalistischer Rhetorik klingt aber deren agitatorische Polemik gegenüber Andersgläubigen, in der die Anwendung von Gewalt zur gottgewollten, verdienstvollen Tat deklariert und der Täter zum Märtyrer erhoben wird. So spricht etwa Bernhard von Clairvaux in seinem flammenden Lob der Templer von "gutem Gewissen«, mit dem »ein Ritter Christi« die Ungläubigen tötet, weil er »nicht ein Menschenmörder, sondern sozusagen ein Mörder der Bosheit« ist. Daher kann er auch ruhig dem Tod entgegensehen. Denn wenn er stirbt »ist er nicht untergegangen, sondern ans Ziel gelangt« (Bernhard von Clairvaux 1990: 
266f.). Nicht häufig, aber doch ab und an melden sich durchaus Zweifel an dieser martialischen Position, etwa wenn Wolfram im Willehalm Gyburc zur Schonung der Heiden aufrufen lässt, weil auch sie Geschöpfe Gottes sind (vgl. Wolfram von Eschenbach 1991: 306,27-309,11).

Und so changiert ein unvoreingenommener interkultureller Dialog mit verschiedensten Formen mittelalterlicher Literatur immer wieder zwischen Befremden, Staunen, Wiedererkennen, Nichtwissen, Vertrautheit und Distanz. Die in der Forschung so selbstverständlichen und hier ja auch benutzten Differenzbegriffe >eigen < und >fremd < beginnen nicht nur unscharf zu werden, sondern entwickeln sich zu eigentlichen Komplementärbegriffen. Das Gegenüber - in unserem Fall der mittelalterliche Text - ist je nach Situation und Kontext der Begegnung entweder ferner oder naher Spiegel (vgl. Tuchman 1987) des eigenen Selbst, ist Teil des Ichs oder eher schwer zu verstehender >Fremdkörper<, kann aber auch beides gleichzeitig sein. Eines aber besitzt der mittelalterliche Text nie: ein identifikatorisches Potenzial, aus dem sich eine eigene, homogene kulturelle Identität linear ableiten ließe.

\section{3. ÄHNLICHKEIt ALS StRUKTURPRINZIP}

Damit zeigt sich aber, dass nicht nur »das Fremde [...] eine relationale Größe ist« (Münkler 2002: 325), sondern, je nachdem welche Kategorien zur Bestimmung herangezogen werden, auch das Eigene. Differenz als »Paradigma der Wissensorganisation « (Bhatti/Kimmich 2015: 8) scheint damit als Erklärung nicht zu genügen. Deshalb setzen Bhatti und Kimmich diesem das »Konzept [...] der kulturellen Ähnlichkeit« entgegen (ebd.: 9). Dabei sei es - so erläutern sie weiter- gerade die in der Forschung bisher gescholtene Vagheit dieses Begriffs, die ihn als »Strukturprinzip« prädestiniert, »nach dem die Entitäten angeordnet sind « (ebd.: 12). Ähnlichkeitsdenken hebt deshalb Unterschiede und Differenzen nicht auf, aber es wendet sich gegen die strikte Dichotomie und Binarität von >eigen « und >fremd <, d.h., es destabilisiert, ja löst »kulturelle Hierarchisierungen « (ebd.: 17) auf und öffnet den Blick auf »geteilte und gemeinsame Geschichte(n) und ihre Verwobenheit ineinander« (ebd.: 19). Die Kategorien >fremd < und >eigen < werden deshalb nicht negiert, aber sie fungieren nicht mehr als auf Differenz angelegte kulturelle Deutungsmuster. Denn wenn der Fokus auf dem Suchen und Erkennen von Ähnlichkeiten liegt, verschwimmen deren Grenzen, ohne deshalb die zeitliche Distanz und/oder die Verschiedenheit des kulturellen Umfelds aus dem Blick zu verlieren. Es entsteht nicht nur eine interkulturelle, sondern eine plurikulturelle Sichtweise, die keine Identifikation einfordert, weil die »Ähnlichkeit in der Diversität« betont wird, nicht das Eigene oder das Einende »trotz Diversität« (Bhatti 2015: 131). Der interkulturelle Dialog mit mittelalterlichen Texten unter diesem Postulat verändert nicht zuletzt das »Wissen um die eigene Kulturalität« und löst damit tendenziell den oben konstatierten Widerspruch auf. Denn der »mentale Abstandsraum« (Gutjahr 2010: 19) zum angeblich >eigenen< Mittelalter erweist sich als genauso groß oder klein wie zu an- 
deren Kulturen. Als zentral gesetzte identitätsstiftende Kategorien wie Sprache, Herkunft, Tradition verlieren sie ihre differenzierende Kraft. Vorstellungen von einer historisch gewachsenen homogenen kulturellen Identität werden zwangsläufig ersetzt durch das Wissen darüber, dass kulturelle Identitäten zu allen Zeiten geprägt waren von interkulturellen Beziehungen, Einflüssen und Vernetzungen, von einem dauernden Changieren, ja z.T. Verschmelzen von Fremdem und Eigenem, von Nähe und Distanz, von »Prozesse[n] der Integration und Desintegration « (Schneidmüller/Seitz 2008: 565f.) und - ganz entscheidend - von dauerndem Wandel. Dessen Komplexität ist Gegenstand kulturgeschichtlicher Forschung, »nicht das Wesen einer Kultur« (Dücker/Müllerburg 2011: 563f.).

Gleichzeitig weist die Beschäftigung mit mittelalterlichen Texten aber auch auf eine Gefahr hin, die dem Begriff >Ähnlichkeit< inhärent ist. Denn auf Ähnlichkeitsdenken basieren mittelalterliche Wissenschaft und mittelalterliches Weltbild, allerdings gerade nicht im plurikulturellen Sinn, sondern ausgehend von einer Norm, von der aus Ähnlichkeiten bzw. Analogien gesucht, aber auch und gerade Abweichungen markiert werden. Mit der Annahme, dass jedes Ding der Welt eine Entsprechung im menschlichen Körper hat, ließ sich der Mensch als Mikrokosmos im Makrokosmos positionieren (vgl. Sollbach 1995). Auch konnten Geschlechterhierarchien eine wissenschaftliche Begründung finden, indem der Mann zur Norm erklärt und die Frau an diesem gemessen wurde, was sie zu einem ähnlichen, aber nicht gleichen Derivat des Mannes machte (vgl. Fietze 1991: 40f.). Genauso konnte eine ganz bestimmte Sprache, ein ganz bestimmtes Aussehen, ein Glaube und eine Ethnie als Norm festgelegt werden, so dass alle Abweichungen davon dieser Norm untergeordnet oder gar als defizitär betrachtet wurden. Mittelalterliche Literatur spielt auf vielfältige Weise mit einer solch >normativen Ähnlichkeit<. Belakane ist eine makellos höfische Dame, nur die normativ gesetzten Kategorien Religion und Ethnie erfüllt sie nicht. Sie ist keine Christin und verfügt nicht über das höfische Ideal eines weißen Körpers, ungeachtet ihrer an die Hoheliedverse - »nigra sum, sed formosa (schwarz bin ich, aber schön) « (Cantica canticorum 1985 1: 5) - gemahnende Schönheit (Wolfram von Eschenbach 1998: 24,1-20 u.ö.; vgl. Salama 2014). Die Kranichmenschen im Herzog Ernst (vgl. 1994) verfügen über ein ideales höfisches Sozialwesen, höfische Kleidung und höfische Lebensformen, fehlende Sprache und Vogelköpfe aber schließen sie aus der menschlichen Gesellschaft aus (vgl. ebd.: vv. 2850-2889, 3057-3089 u. 3151-3159). Die Heiden in christlichen Heldenepen sind kampfstark, höfischen Rittern adäquat in Ausstattung und ritterlichen Kampfformen, ihr nicht christlicher Glaube aber wird zu »einem zentralen Bezugspunkt für Praktiken der Grenzziehung « (Dücker/Müllerburg 2011: 568). (Fremd-)Sprachliche Kompetenz, wie sie etwa Willehalm besitzt oder der heidnische, Französisch sprechende Burggraf im Guoten Gêrhart (vgl. Rudolf von Ems 1971: vv. 1350-1352; Schumacher 2010: 53), fördert dabei aber wenigstens das Gelingen interkultureller Kommunikation.

Bevor man nun vorschnell diese Normierungen als veraltet, eben stypisch mittelalterlich< abtut, gilt es, sehr genau zu überlegen, ob nicht bis heute ein interkultureller Dialog oder interkulturelle Begegnungen so häufig scheitern, 
weil - in der Regel wohl unbewusst - das Ich sich selbst genauso wie im Mittelalter als Norm begreift, von dem ausgehend dann Ähnlichkeit nichts anderes ist als das Eigene und Unähnliches nichts anderes als das Fremde. Ähnlichkeit als Strukturprinzip zu begreifen zwingt dagegen zur kritischen Hinterfragung oder mehr noch zur Verwerfung dieser Positionierungen und schärft den Blick für interkulturelle Beziehungsnetze auch und gerade in mittelalterlichen Texten. Denn es wird dann deutlich sichtbar, dass interkulturelle Begegnungen das Übliche, nicht die Ausnahme sind. Konflikte können genauso daraus entstehen wie Wissenserweiterungen, gegenseitige kulturelle Beeinflussungen, gelingende oder auch scheiternde interkulturelle Dialoge, Exklusion und Inklusion. D.h., es finden sich genau die Themen, die auch im Gegenwartsdiskurs zentral sind, eine prozesshaft verstandene Interkulturalitätsforschung beschäftigen und im Konzept der Ähnlichkeit beschreibbar werden. Denn Ähnlichkeit - dies sei noch einmal dezidiert betont - ist sowohl dem vermeintlich Eigenen wie dem vermeintlich Fremden inhärent.

\section{REsÜMEe}

Auch wenn manches hier nur verkürzt angesprochen und diskutiert werden konnte, so lassen sich abschließend doch erste, zumindest vorläufige Antworten auf die am Anfang gestellten Fragen geben:

1. Interkulturelle Zugänge zu mittelalterlich-deutschsprachiger Literatur zeigen, dass das Mittelalter weder finster noch golden, weder besser noch schlechter, weder idealer noch rückständiger, weder grausamer noch friedfertiger war als andere Zeiten und Kulturen. Sie führen zur Erkenntnis, dass interkulturelle Kontakte Sprache, Literatur, Politik und Lebensformen in hohem Maß beeinflussen, ein eigentliches Nationalbewusstsein oder gar ein Nationalstaat aber dem mittelalterlichen Menschen gänzlich fremd war. D.h., interkulturelle Zugänge setzen eindimensionalen, sich hermetisch abschottenden Setzungen eines wie auch immer gearteten nationalen Mittelalters die immanente Interkulturalität des Mittelalters entgegen, die jede Verabsolutierung verbietet und damit Vorstellungen einer sich entwickelnden, auf Tradition beruhenden, homogenen Kultur als meist politisch motivierte Konstrukte späterer Jahrhunderte entlarvt.

2. Während Alterität das Fremde der mittelalterlichen Texte betont und damit einen Blick fördert, der diese als exotisch taxiert und/oder als unterentwickelt abqualifiziert, fordert ein interkultureller Dialog zum genauen Zuhören heraus, d.h. zum vorurteilsfreien Nachforschen nach Kontexten, Zusammenhängen, Denkformen und Grundlagen des Weltverständnisses, aus denen heraus die Texte entstanden sind. Dies gilt umso mehr, wenn Interkulturalität als nie abgeschlossener Prozess verstanden wird, mithin jede Arbeit an den Texten keinem starren Paradigma folgt, sondern immer veränderbar bleibt. Und wenn dann Ähnlichkeit als Strukturprinzip den 
Analysen unterlegt wird, lösen sich Differenzbegriffe sowie starre Grenzen zwischen dem Eigenen und dem Fremden, zwischen Nähe und Distanz, zwischen heute und früher auf. Stattdessen eröffnet sich ein Panoptikum interkultureller Kontakte, Vernetzungen und Grenzüberschreitungen sowie vielfältige Formen der Exklusion wie Inklusion. Mittelalterliche literarische Stoffe erweisen sich dabei in überaus vielfältiger Weise als kulturübergreifend und können durchaus - wie Herweg forciert formuliert - als »Migrationsliteratur« (Herweg 2017: 16) bezeichnet werden.

3. Wenn man »Fragen, die unser Leben in Atem halten« (Gurjewitsch 1993: 12), mittelalterlichen Texten stellt, dann bedeutet dies keineswegs nur, dass man neue Erkenntnisse und Antworten darauf gewinnt, sondern auch, dass man Analyseinstrumente erprobt und Wissen generiert, die mit und an dem Postulat des interkulturellen Diskurses der Gegenwart gemessen, hinterfragt und möglicherweise sogar verworfen werden. Ich würde deswegen nicht so weit gehen, »die Beschäftigung mit der Literatur des Mittelalters« als »eine unverzichtbare Grundlage « zu behaupten, um mit »Eigenem und Fremdem zurechtzukommen« (Kasten/Bachorski/Kugler 1997: 68). Aber ohne den historischen Blick im Interkulturalitätsdiskurs und ohne hermeneutische Fähigkeiten, wie sie an mittelalterlicher Literatur besonders intensiv geschult werden können, ist die Gefahr groß, immer wieder vor allem das Trennende, nicht das Gemeinsame der Kulturen zu erkennen. Und das ist keineswegs eine Legitimationsstrategie der Mediävistik, wie oft behauptet wird (u.a. von Braun 2013: 8), sondern ein Plädoyer dafür, dass der synchrone Blick auf kulturelle Identitäten des Diachronen bedarf, um Ähnlichkeiten, Konstanten und Wandel als zu ihnen gehörig, mehr noch: als für sie konstitutiv zu erkennen.

\section{LITERATUR}

Becker, Anja/Mohr, Jan (Hg.; 2012): Alterität als Leitkonzept für historisches Interpretieren. Berlin.

Bernhard von Clairvaux (1990): In laude militum Templi. In: Ders.: Sämtliche Werke, Bd. 1. Hg. v. Gerhard B. Winkler, Innsbruck, S. 268-321; online unter: http://www. glaubensstimme.de/doku.php?id=autoren:b:bernhard_von_clairvaux:bernhardtempelritter [Stand: 1.9.2018].

Bhatti, Anil (2015): Heterogenität, Homogenität, Ähnlichkeit. In: Zeitschrift für interkulturelle Germanistik 6, H. 1, S. 119-133.

Ders./Kimmich, Dorothee (Hg.; 2015): Ähnlichkeit. Ein kulturtheoretisches Paradigma. Paderborn.

Bendheim, Amelie (2017): Wechselrahmen. Medienhistorische Fallstudien zum Romananfang des 13. Jahrhunderts. Heidelberg.

Braun, Manuel (2013): Alterität als germanistisch-mediävistische Kategorie: Kritik und Korrektiv. In: Ders. (Hg.): Wie anders war das Mittelalter? Fragen an das Konzept der Alterität. Göttingen, S. 7-38. 
Brinker-von der Heyde, Claudia (2007): Die literarische Welt des Mittelalters. Darmstadt.

Cantica Canticorum Salomonis (1985). In: Biblia Sacra iuxta Vulgatam Clementiam. Madrid, S. 614-618.

Dücker, Julia/ Müllerburg, Marcel (2011): Bilanz eines Aufbruchs. In: Michael Borgolte (Hg.): Integration und Desintegration der Kulturen im europäischen Mittelalter. Berlin, S. 561-568.

Fietze, Katharina (1991): Spiegel der Vernunft. Theorien zum Menschsein der Frau in der Anthropologie des 15. Jahrhunderts. Paderborn.

Flechsig, Karl-Heinz (2001): Auf dem Weg zur interkulturellen Gesellschaft. Konzepte zur interkulturellen Arbeit. In: Petra-Kelly-Stiftung/Bayerisches Bildungswerk für Demokratie und Ökologie in der Heinrich-Böll-Stiftung e.V. Nürnberg (Hg.): Auf dem Weg zur "interkulturellen " Gesellschaft. Trainings und Konzepte zur interkulturellen Arbeit. Tagungsdokumentation, S. 1-29; online unter: https://www.petra kellystiftung.de/fileadmin/user_upload/newsartikel/PDF_Dokus/Interkulturelle_ Gesellschaft.pdf [Stand: 1.9.2018].

Földes, Csaba (2007): Interkulturelle Kommunikation. Positionen zu Forschungsfragen, Methoden und Perspektiven. Wien.

Gurjewitsch, Aaron J. (1993): Stimmen des Mittelalters. Fragen von heute. Frankfurt a.M.

Gutjahr, Ortrud (2010): Interkulturalität als Forschungsparadigma der Literaturwissenschaft. Von den Theoriedebatten zur Analyse kultureller Tiefensemantiken. In: Dieter Heimböckel/Irmgard Honnef-Becker/Georg Mein/Heinz Sieburg (Hg.): Zwischen Provokation und Usurpation. Interkulturalität als (un)vollendetes Projekt der Literatur- und Sprachwissenschaften. München, S. 17-39.

Guzzoni, Ute (2012): erstaunlich und fremd. Erfahrungen und Reflexionen. Freiburg i.Br. Hart-Nibbrig, Christiaan L. (1999): Zwischen den Kulturen: Kulturwissenschaft als Grenzwissenschaft. In: Johannes Anderegg/Edith Anna Kunz (Hg.): Kulturwissenschaften. Positionen und Perspektiven. Bielefeld, S. 93-104.

Hartmann von Aue (2015): Die Klage. Hg. v. Kurt Gärtner. Berlin.

Heimböckel, Dieter/Weinberg, Manfred (2014): Interkulturalität als Projekt. In: Zeitschrift für interkulturelle Germanistik 5, H. 2, S. 119-143.

Herder, Johann Gottfried (1878): Kritische Wälder. In: Ders.: Sämtliche Werke. Hg. v. Bernhard Suphan. Bd. 3. Berlin.

Herder, Johann Gottfried (1891): Auszug aus einem Briefwechsel über Oßian und die Lieder alter Völker. In: Ders.: Sämtliche Werke. Hg. v. Bernhard Suphan. Bd. 5. Berlin, S. 159-207.

Herweg, Mathias (2017): Alterität und Kontinuität. Vom interkulturellen Potential der germanistischen Mediävistik. In: Zeitschrift für interkulturelle Germanistik 8, H. 1, S. 11-23.

Herzog Ernst. Ein mittelalterliches Abenteuer. Mittelhochdeutsch/Neuhochdeutsch (1994). In der mittelhochdeutschen Fassung B nach der Ausg. v. Karl Bartsch mit den Bruchstücken der Fassung A. Übers., mit Anmerkungen u. einem Nachwort versehen v. Bernhard Sowinski. Stuttgart. 
Herzog, Roman (1998): Rede von Bundespräsident Roman Herzog anläßlich der Verleihung der Goethe-Medaillen; online unter: http://www.bundespraesident.de/ SharedDocs/Reden/DE/Roman-Herzog/Reden/1998/03/19980322_Rede.html [Stand: 1.9.2018].

Huntington, Samuel P. ( $\left.{ }^{6} 1997\right)$ : Der Kampf der Kulturen. The clash of civilizations. Die Neugestaltung der Weltpolitik im 21. Jahrhundert. München.

Illich, Ivan (1991): Im Weinberg des Textes. Als das Schriftbild der Moderne entstand. Ein Kommentar zu Hugos "Didascalicon". Frankfurt a.M.

Kasten, Ingrid/Bachorski, Hans Jürgen/Kugler, Hartmut (1997): Das eigene Fremde. Mediävistik und interkulturelle، Kompetenz. In: Mitteilungen des Deutschen Germanistenverbandes 44, H. 4, S. 66-74.

Leggewie, Claus/Zifonun, Dariuš (2010): Was heißt Interkulturalität? In: Zeitschrift für interkulturelle Germanistik 1, H. 1, S. 11-31.

Marschall, Wolfgang (1999): Wozu die Kulturwissenschaften da sind. In: Johannes Anderegg/Edith Anna Kunz (Hg.): Kulturwissenschaften: Positionen und Perspektiven. Bielefeld, S. 19-29.

Müller, Ulrich (2009): Von den ,falschen Freunden،. Das Übersetzen aus alten Sprachen (Mittelhochdeutsch) als interkulturelles Problem. In: Ernest W.B. Hess-Lüttich u.a. (Hg.): Translation und Transgression. Interkulturelle Aspekte der Übersetzung(swissenschaft). Frankfurt a.M./ New York, S. 213-229.

Münkler, Marina (2002): Alterität und Interkulturalität (ÄdL). In: Claudia Benthien/ Hans Rudolf Velten (Hg.): Germanistik als Kulturwissenschaft. Eine Einführung in neue Theoriekonzepte. Reinbek b. Hamburg, S. 323-344.

Niehaus, Michael (2010): Interkulturelle Dinge. In: Zeitschrift für interkulturelle Germanistik 1, H. 1, S. 33-48.

Novalis (1986): Fragmente und Studien. Die Christenheit oder Europa. Hg. v. Carl Paschek. Stuttgart.

Oexle, Otto Gerhard (1997): Die Moderne und ihr Mittelalter. Eine folgenreiche Problemgeschichte. In: Peter Segl (Hg.): Mittelalter und Moderne. Sigmaringen, S. 307-364.

Ridder, Daniela De/Jorzik, Bettina (Hg.; 2012): Vielfalt gestalten. Kernelemente eines Diversity-Audits für Hochschulen. Essen; online unter: https://www.uni-hamburg. de/gleichstellung/diversity/box-diversity-dimensionen.html [Stand: 1.9.2018].

Rudolf von Ems (1996): Der guote Gêrhart. Hg. v. John A. Asher. Tübingen.

Salama, Dina Aboul Fotouh (2014): Formen und Funktionen orientalischer Körper im ,Parzival، Wolframs von Eschenbach. In: Archiv für das Studium der neueren Sprachen und Literaturen 251, S. 1-34.

Schlegel, August Wilhelm (1965): Geschichte der romantischen Literatur. Stuttgart.

Schlesier, Renate: Das Staunen ist der Anfang der Anthropologie. In: Hartmut Böhme/ Klaus Scherpe (Hg.): Literatur und Kulturwissenschaften. Positionen, Theorien, Modelle. Reinbek b. Hamburg, S. 47-59.

Schmitz, Walter (1991): Das Eigene als das Fremde. "Interkulturalität" als Forschungsparadigma für die Germanistik. In: Bernd Thum/Gonthier-Louis Fink (Hg.): Praxis interkultureller Germanistik. Forschung-Bildung-Politik. Stuttgart/Weimar, S. 171175. 
Schneidmüller, Bernd / Seitz, Annette (2008): Transkulturelle Mediävistik - ein Schlusswort. In: Michael Borgolte/Juliane Schiel/Bernd Schneidmüller/Annette Seitz (Hg.): Mittelalter im Labor. Die Mediävistik testet Wege zu einer transkulturellen Europawissenschaft. Berlin, S. 557-566.

Schumacher, Meinolf (2010): Toleranz, Kaufmannsgeist und Heiligkeit im Kulturkontakt mit den 'Heiden. Die mittelhochdeutsche Erzählung Der guote Gêrhart von Rudolf von Ems. In: Zeitschrift für interkulturelle Germanistik 1, H. 1, S. 49-58.

Senghaas, Dieter (1998): Zivilisierung wider Willen. Der Konflikt der Kulturen mit sich selbst. Frankfurt a.M.

Sieburg, Heinz (2011): Plädoyer für eine interkulturelle Mediävistik. In: Zeitschrift für interkulturelle Germanistik 2, H. 1, S. 11-26.

Sollbach, Gerhard E. (1995): Die mittelalterliche Lehre vom Mikrokosmos und Makrokosmos. Hamburg.

Stanitzek, Georg (1987): Der Projektmacher. Projektionen auf eine ıunmöglicher moderne Kategorie. In: Ästhetik \& Kommunikation 17, H. 65/66, S. 135-146.

Thomasin von Zerklaere (1965): Der Wälsche Gast. Berlin.

Tuchman, Barbara (1987): Der ferne Spiegel. Das dramatische 14. Jahrhundert. Düsseldorf.

Weisgerber, Leo (1966): Vorteile und Gefahren der Zweisprachigkeit. In: Wirkendes Wort 16 , H. 2, S. 273-289.

Welsch, Wolfgang (2010): Was ist eigentlich Transkulturalität? In: Lucyna Darowska/ Thomas Lüttenberg/Claudia Machold (Hg.): Hochschule als transkultureller Raum? Kultur, Bildung und Differenz in der Universität. Kultur und soziale Praxis. Bielefeld, S. 39-66; online unter: http://docplayer.org/44016-Wolfgang-welsch-fried rich-schiller-universitaet-jena-was-ist-eigentlich-transkulturalitaet.html [Stand: 1.9.2018].

Wenzel, Horst (1995): Hören und Sehen, Schrift und Bild. Kultur und Gedächtnis im Mittelalter. München.

Wirnt von Grafenberg (2005): Wigalois. Text - Übersetzung - Stellenkommentar. Text der Ausgabe von J.M.N. Kapteyn. Übers., erl. u. mit einem Nachwort vers. v. Sabine Seelbach u. Ulrich Seelbach. Berlin.

Wolfram von Eschenbach (1991): Willehalm. Nach der Handschrift 857 der Stiftsbibliothek St. Gallen. Mhd. Text. Hg., übers. u. komm. v. Joachim Heinzle. Frankfurt a.M.

Ders. (1998): Parzival. Mittelhochdeutscher Text nach der 6. Ausg. v. Karl Lachmann. Übers. v. Peter Knecht. Einführung zum Text v. Bernd Schirok. Berlin.

Yousefi, Hamid Reza/ Braun, Ina (2011): Interkulturalität. Eine interdisziplinäre Einführung. Darmstadt. 